\title{
Ser agente de salud y el cuidado humanitario. Contribuyendo a su transformación
}

\section{Being a health agent and the humanitarian care. Con- tributing to its transformation}

Lic. Enf. Susana Salas Segura•

Mtra. Rosa A. Zárate.•

Dr. Rafael Chávez Domínguez..•

Resumen

El cuidado humanitario es un acto del ser, susceptible de evolucionar y conformar una unidad de cuidado a la salud con calidad para todos y requiere también de cuidado para no perder su valor de sentido positivo. Conciliar teoría y práctica no es simple, sino complejo. En este artículo se propone atender la relación Agente de Salud-Paciente por ser universal y congruente con el cambio. El enfoque epistemológico puntualiza lo positivo, lo acumulado en la historia no puede desaparecer del presente. La respuesta es integración, hacer coincidir las ciencias formales, con las sociales, humanas y la tecnología. El apoyo cultural es vital, la educación es sólo parte y convoca a construir el nuevo paradigma; al invocarlo se superan las acciones ineficaces. El acto preventivo es a todos los niveles, sea del hospital, la escuela, la comunidad el hogar y la comunidad entera. La Enfermería del futuro, con su desarrollo teórico, contribuye al cambio, del cuidado reduccionista al integral y humanitario. Se propone reforzar la atención al desempeño del Agente de Salud y orientarlo con la disposición Humanitaria. El tema profundiza en el conocimiento del cuidado y le concierne a todo profesional de la salud.

\section{Palabras}

Clave:

Cuidado humanitario, Filosofía y Enfermería,

Epistemología

y Enfermería,

Agente de Salud

-Ex Directora de la ENEO UNAM

..Profesora de TC de la ENEO UNAM. Coordinadora de Investigación

...Jefe del Departamento de Epidemiología del Instituto Nacional de Cardiología Ignacio Chávez

correo-e rchavez@cardiologia.org.mx

RECIBIDO: 13 JULIO 2011

ENVIADO: 18 Julio 2011

ACEPTAdo: 5 SEPTIEMBRE 2011 


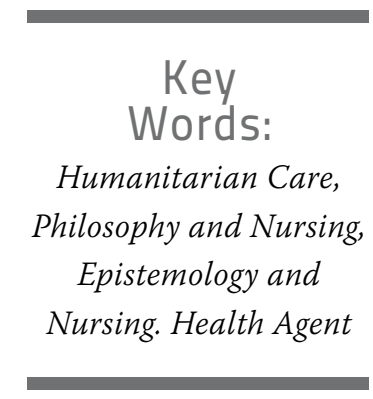

Abstract

The humanitarian care is an act, the being who may evolve and transformed into a quality unit for everybody's health care. Requires attention, good care, and not to lose the sense of positive value. Must reconcile theory and practice which is not so simple, it is rather complex. We propose to address the Relationship of Health Agent - Patient to a universal approach consistent with the changing nature. The epistemological approach points out the positive sense. The history accumulated knowledge cannot fail at the present time. The answer is integration, to combine formal sciences with social and human, as well as technology. The cultural support is vital for education and is part of it, calls for the build the new paradigm. To invoke it is sufficient to avoid ineffectiveness. The preventive act is at all levels, whether in hospital, school, community, home and the entire community. The future nursing, with the care models, should contribute to shift from the reductionist care to the humanitarian care. Let us reinforce the Health Agent performance and guide it towards a Humanitarian Care. The issue analyzes the care knowledge and is of every health server concern.

\section{INTRODUCCIÓN}

El cuidado humanitario es la idea de construir juntos un modelo unitario, algo por conocer con más intimidad a partir de apreciar lo común de los cuidados particulares en la diversidad y su inclusión en el cuidado a la Salud. También es apoyo para no perder el sentido positivo en la conducta y concilia la teoría y la práctica en bien de la prevención y la salud. Integrado en la relación Agente de Salud- Paciente/Persona (AS-P) es más universal y congruente con el proceso de cambio. El enfoque epistemológico puntualiza lo acumulado por la historia. Lo pasado y útil no puede dejar de estar presente. No obstante lo complejo de las áreas y disciplinas que intervienen, su integración puede descubrir soluciones concretas y ser la llave maestra para compaginar las ciencias sociales, las ciencias humanas y las ciencias duras, con la participación de la tecnología, en apoyo a la formación cultural amplia, donde la educación es sólo una parte y convoca a construir el paradigma común de las creencias del dominio popular. Es necesario invocarlo para superar las acciones ineficaces. La acciones preventivas en todos los niveles de atención ya sea en el hospital, la escuela, la comunidad el hogar, la cama de la unidad de cuidados intensivos y los conocimientos, habilidades, actitudes y valores de la Enfermería del futuro son el complemento necesario para cambiar el paradigma del cuidado reduccionista por el cuidado humanitario. Esto en síntesis es lo que se pretende transmitir en este documento; tomar en cuenta el amplio ámbito del Agente de salud dedicado vocacional y profesionalmente al Cuidado Humanitario.

\section{Perspectiva del cuidado}

El asunto es vital para los fines de procurar la salud. Obviamente atañe a todos, porque a todos nos debe interesar cuidar la salud; pero especialmente a los profesionales de la medicina, la enfermería y otros, incluyendo a los técnicos relacionados con el desempeño formal de los cuidados de salud. El marco conceptual alude al Agente de Salud como la persona en actitud de ayudar a otras personas proporcionando algún cuidado; y lo relaciona con el Cuidado Humanitario, como el conjunto de cuidados de salud, dentro de una visión unitaria y universal.

Mientras el cuidado es determinado por la necesidad del enfermo, la ayuda la define la persona que ayuda al integrar sus facultades intelectuales, corporales y volitivas. El requisito de estos componentes se comprende mejor al contrastarlos, pues es inconcebible que un Agente de Salud capaz, carezca de voluntad, "un cuerpo sin alma"; y viceversa, un Agente de Salud con voluntad, pero incapaz, "una alma sin cuerpo" Lo real y deseable sería ponderar y dosificar asertivamente estos valores. El concepto de humanitario contiene el valor supremo del acto de cuidar, precisa de la 
dualidad que conforman a la persona: cuerpo y alma. Se entiende que para ejercer los cuidados se requiere preparación, y se han de realizar mediante la presencia corporal del Ser; pero además requiere voluntad que sale de su conciencia, por lo que también reclama encauzar su vocación.

El conjunto de cuidados, actos diversos en personas diferentes, es un gran cúmulo de actos, un núcleo de acciones. Propicia la visión unitaria para descubrir en ello los valores inherentes, que se hacen presentes por lo que son y por lo que representan de su entorno. El cuidado humanitario, visto como conjunto, brinda la oportunidad de plantear y reflexionar sobre varios otros conceptos que cumplen la función de identificar y proponer soluciones prácticas; no una para todos sino una para cada caso.

El punto de vista integral es un marco de referencia para apreciar lo innovador, pues abarca todos los procedimientos de atención a la salud que confluyen en el acto de curar y prevenir. No todo es combatir a la enfermedad, sino también atender a sus antecedentes $o$ los factores de riesgo. ${ }^{1}$ En la lucha por el bienestar y la salud, también concuerdan los cuidados por la vida y desarrollo social y humano. Como insistía la enfermera y antropóloga Marie Francoise Colliere, promover la vida. ${ }^{2}$

El reto de la atención comunitaria hoy día, se justifica porque predominan las enfermedades crónicas, cardiovasculares, metabólicas, degenerativas y cáncer que lo demandan. La idea innovadora es hacer más comprensivo el principio de la tríada ecológica: (agen- te etiológico; huésped-individuocomunidad; y ambiente), que han funcionado en las enfermedades transmisibles. Facilita penetrar a las raíces dispersas de los problemas inherentes, en todos los sectores de la población y, al mismo tiempo, ofrece la posibilidad de teorizar, contemplar y disertar en cosas prácticas. El enfoque puede ayudar a movilizar la voluntad común, que es un imperativo moral.

\section{El enfoque humanitario}

Se hace también, en este artículo, el ejercicio de mirarlo como enfoque humanitario, para recoger directamente, de su ambiente, el interés de la persona por ayudar, no sólo como caso aislado de enfermedad, o procedimientos y tecnologías aplicables a casos concretos, sino de revisar ideas, opiniones, diversidad de criterios y hacer comentarios pertinentes.

El cuidado humanitario se apoya en un valor jerárquico por encima de los cuidados individuales. Es aplicable no sólo a pacientes, sino a comunidades, con el potencial de intervenir con las personas en la transición de la salud - enfermedad. Los conocimientos que lo avalan se han originado en hechos científicos sólidos, elaborados con detallados estudios epidemiológicos y cimentados en prolongadas observaciones comunitarias, en su mayoría generados en la última mitad del Siglo xx y ampliamente divulgados, ya forman parte del acervo de conocimientos de la Medicina, la Enfermería Familiar y Comunitaria; sin embargo, hace falta zanjar la brecha entre lo científico y teórico con lo práctico, precisamente con la orientación apropiada de integrarlo al cuidado.
Preocupa encauzar el enfoque crítico a los grandes problemas de salud y darles soluciones. Se ha visto que los cuidados no corresponden a la calidad de atención esperada. El enfoque puede facilitar hacerlos apropiadamente con el conocimiento de sus relaciones, vínculos y conexiones con el entorno. Así mismo visualizar el común denominador y orientarlo a la protección de las personas con enfermedades, justificando el enfoque de los factores condicionantes. El modo unitario los aprecia mejor por su índole, sin perder la polarización, a fin de implementar un control más radical dirigido a resolver las inconsistencias propias de problemas prácticos, como Enfermedades Cardiovasculares, Diabetes Mellitus, Enfermedades metabólicas, Neuroencefalopatías, Alzheimer, Cáncer y otros padecimientos sistémicos.

El reto implica crear estrategias, implementar acciones apropiadas, evitar condiciones facilitadoras; como adicciones y tóxicos; pero también actuar sobre las condiciones adversas derivadas de la multicausalidad arraigada en hábitos dañinos y estilos de vida equivocados. Las áreas de acción se ubican más allá de los linderos de la medicina y la enfermería, razón por lo cual no es insensato pensar que rebasen las áreas del Sector Salud, para situarse finalmente en el campo de lo social y cultural de la comunidad global, y no por ello hay que perder su enfoque de interés último por la salud y el bienestar.

Las enfermedades crónicas y no transmisibles, antes conocidas como incurables, hoy son suficientemente controlables atendiendo a 
los Factores de Riesgo (FR). Aunque la meta parezca cuesta arriba por la pretensión de cubrir idealmente a toda la comunidad, en realidad incluye medidas eficientes de prevención, sustentadas por investigaciones científicas basadas en evidencia y aceptadas como estrategia poblacional. ${ }^{3}$

La atención a las personas con enfermedades crónicas y otras condiciones mediante los cuidados pertinentes, son una necesidad en varios países del mundo, incluye a los de mayor desarrollo económico, pues sus frecuencias exceden a la prevalencia esperada. Más aún, algunas comunidades, paradójicamente, han incrementado su proporción a partir de cuando iniciaron intervenciones masivas. En otros ámbitos, la novedad es la desigualdad acentuada, atribuible no sólo a la variabilidad biológica de la enfermedad, sino a los factores condicionantes $^{4,5}$ Todo indica que no es suficiente con hacer lo que se sabe, hay que saber más y llevarlo a la práctica para cuidar mejor.

\section{Unidad, universalidad y las bondades del planteamiento}

El enfoque unitario permite apreciar el problema de fondo en el mundo. No hacen falta estudios especiales para reconocer que tiene vinculación con el relativismo cultural, ${ }^{6}$ cognitivo y moral. Se trata de una actitud ampliamente difundida y lamentablemente resultado de crecer sin tomar conciencia de los valores universales, como el concepto de respeto, propio y ajeno. De no atender a este punto, no sólo se perjudica la población, sino que se dilapida el bienestar y la salud. Lo lógico en reciprocidad, es nutrirse de valores y entrar al humanitaris- mo por el beneficio de quienes más lo necesitan las personas sujeto de nuestro cuidado.

Los turbulentos problemas sociales que se viven hoy, expresión de las crisis sociales, están constantemente en los medios noticiosos, y son explicables por ese relajamiento de valores. El daño ocurre dentro de los individuos y entre la población, el blanco lo sufren los sectores más vulnerables vinculados con la salud y el bienestar, con la enseñanza y aprendizaje; justicia y seguridad; trabajo, producción finanzas y economía. El resultado se presenta de diversas formas, en muchas y diferentes comunidades por todo el planeta.

Lo humanitario, también es tema que vincula la salud con la conducta individual, en unión común con la voluntad o comunión. Los indicadores del daño a la salud, revelan tasas elevadas de mortalidad, morbilidad e incapacidad y se les responsabiliza del deterioro nacional y global. Hay que advertir que el principio causal aplicado ha sido inapropiado, por darle mayor crédito a la primera instancia causal y omitiendo la participación de otros factores. La omisión refleja que las cosas han sido hechas a medias y explica porqué las amplias campañas preventivas no dan el resultado que se espera.

En la literatura de la salud, así como en otro tipo de documentos abundan las estadísticas, datos, cifras, proporciones elocuentes e interpretaciones epidemiológicas que avalan su verosimilitud, ponen en evidencia que la carga asistencial desborda la capacidad instalada y los recursos disponibles. El resultado global es aún más desfavorable, al incluir el complejo genético y metabólico, el común denominador de las enfermedades crónicas degenerativas. Con el enfoque unitario resaltan los hechos, señales de algo más por descubrir y atender. Tal y como se ha comunicado que los suaves cambios cuantitativos determinan drásticas variaciones cualitativas, que advierte $\mathrm{K}$ Popper ${ }^{7} \mathrm{con} \mathrm{su}$ cambio de paradigma y lo anuncia como revolución científica T Kuhn. ${ }^{8}$ Entonces aparecen las nuevas verdades, la nueva ciencia. ${ }^{9}$ Así que lo unitario y holístico puede orientar a encontrar el potencial humanitario.

\section{La dinámica del cambio.}

El avance científico y tecnológico, rápido y significativo del siglo pasado, logrado gracias a un impulso armonioso y universal, contribuyó a que se desarrollaran procedimientos específicos para intervenir, prevenir, aliviar y rehabilitar gran número de personas con variedad de enfermedades en sus diversas etapas evolutivas. Desde entonces, se han multiplicado, refinado, renovado y hoy alcanzan un sorprendente grado de perfección. Consecuentemente hay que reclutar más recursos humanos de calidad, con alto grado de especialización y renovada capacitación; es decir, más Agentes de Salud. Por ello ha habido necesidad de crear y diseminar especialidades y subespecialidades, capacitar personal de la más variada índole insistiendo en el desempeño de calidad. Parafraseando a MF Colliere el buen logro de los cuidados se consigue como una cadena de favores, en donde la ruptura de un eslabón lo malogra todo.

Los profesionales: enfermeras, médicos, ingenieros, biólogos y técnicos, reclaman más recursos técnicos y económicos, en garantía 
de esa calidad, rapidez, precisión y seguridad. Es un efecto colateral e indeseable, ha aparecido como explosión de costos y encarecimiento de servicios vitales. Resulta paradójico que de intentar un bien, se consigue un mal, que no sólo sufre la población con mayores necesidades y en condiciones de marginación, sino todo mundo. Esta complicación no deseada, debe ser superada, ya que los cuidados creados para hacer un bien, dejan de serlo al tener que declinarse por no ser asequibles.

En la población, los nuevos avances tampoco han logrado abatir significativamente los indicadores. El mérito de mejorar la esperanza de vida se diluye ante la desigualdad reinante. Aquí, la visión unitaria descubre fisuras y advierte la necesidad de plantear más extensamente los proyectos para frenar el daño real. Se entiende que la respuesta al reto ha de ser radical y surge la idea de caracterizarla razonable y claramente. La imagen que se busca no se encontraría de otro modo.

La labor es ineludible teórica, de inteligencia y de marcha lenta y difícil, por la vinculación con las áreas sociales, económicas y políticas, a donde no falta controversia. Sin embargo, hay que hacerlo, porque no hay buena práctica sin una buena teoría. ${ }^{10}$ Tampoco cabe esperar a que el producto surja espontáneamente de una persona $\mathrm{o}$ grupo, sino más bien de la participación común y buena voluntad de todos. Entonces adquieren valor los consensos, siempre y cuando se acompañen de concordia de ideas y concilio de criterios. Como sea, encontrar la razón humanitaria es un asunto que pasa de ser importante a urgente. Parte del todo es hallar la justificación racional que explique las cosas, descubrir las interrelaciones de las ideas, encontrar las realidades sensibles y desembocar en una implementación sustentable. Pero compaginar este material teórico referencial, tampoco es suficiente, no basta con sólo pensar, hay que actuar, ensayar, hacer investigaciones, atesorar experiencias, interpretarlas apropiadamente y aplicar sus resultados.

La reflexión crítica necesita calma, tranquilidad, estar libre de conflictos de valor y edificar el conocimiento a partir de sus múltiples y diversos fundamentos. Una condición necesaria es el buen uso de los principios de razonamiento, pues se buscan propuestas concretas y congruentes, explicaciones plausibles, que satisfagan la diversidad y los momentos cruciales de la vida real. Vale la pena aquí, recordar las enseñanzas de Juan Pablo II, dignas de consideración y muy relevantes con el tema "ante una difundida cultura de indiferencia $y$, a veces, de desprecio a la vida, y ante la búsqueda inescrupulosa de predominio por parte de algunos sobre otros, con la consiguiente marginación del pobre y los débiles, hoy es más necesario que nunca ofrecer sólidos criterios, para que el ejercicio del poder en el mundo de la salud, esté en todas la circunstancias al servicio de la dignidad de la persona humana y del bien común". Mismo aserto presente en la Carta Encíclica de Benedicto XVI sobre el desarrollo humano integral. ${ }^{11}$

\section{Revisando la estructura del conocimiento}

En apoyo a la idea unitaria y para fomentar el desarrollo de esa conciencia - metanoia, ${ }^{12}$ que le caiga el veinte, ${ }^{\mathrm{a}}$ es deseable aclarar el punto de convergencia de los cuidados de la salud y pertinente asumir que el cuidado humanitario es un nuevo concepto y para conocerlo, procede seguir el esquema lógico tradicional, analizar sus principios, medios $y$ fines. ${ }^{13}$

\section{Según los principios.}

En el proceso de rastrear un nuevo conocimiento, no resulta igual aplicar un principio que otro, pese a tener los mismos propósitos o fines. Los cuidados particulares engloban a los cuidados generales de salud, pero al cambiar de principio el resultado es diferente. Los principios que conciernen a este tema son: reduccionismo, que facilita llegar al conocimiento especializado, gana en comprensión y adquiere profundidad; pero tiende a perder extensión y con ello disminuye la sensibilidad a los valores universales. Su sentido negativo es perder orientación y confundir los medios con los fines. En cambio, interdisciplinariedad, es comprensión, gana en extensión y permite el aprecio de los valores y no pierde su orientación alineada con fines humanitarios. También está de por medio el principio de orientación, que trasciende en los momentos cruciales de una investigación. Otro principio imprescindible es el de causalidad, por su relación con la validez del conocimiento científico. Consecuentemente, el planteamiento unitario cumple con su arranque al completar el concilio de sus principios: visión holística, comprensiva, orientadora y de validez causal.

a Expresión coloquial mexicana de la época del teléfono público de moneda. 
Según los medios. Agrupados como: a) cuidados, b) actores y c).escenarios.

a)- Los cuidados o actos de cuidar para conservar la salud, son producto de quienes los desempeñan. Lo primero que se advierte es la cambiante terminología, polisemia que distorsiona la comunicación, propicia infructuosas controversias y vale la pena aclarar. En realidad mantienen el mismo significado semántico: curar, cuidar, medicar, atender y asistir. Curar se refiere a procurar los cuidados de vida y tiene que ver, tanto con la salud, como con la enfermedad. Implica ponerle atención, velar, asistir, aplicar el pensamiento, vigilar, tratar, procurar y proteger. Cuidado tiene su raíz en el latín cogitar y significa pensar, preocuparse, interesarse, tenerle afecto, importarle, protegerlo, comprenderlo, tener cautela, celo y responsabilidad. Cuidar invoca atender a la causa, raíz del asunto. Humanamente, implica ayudar al prójimo a crecer, a realizarse como persona y en la comunidad apoyar su desarrollo. En la forma antigua del latín se ligaba al amor y la amistad, con la actitud de cuidado, desvelo, preocupación e inquietud por la persona amada u objeto de estimación. Implica la actitud espontánea de responsabilidad con el prójimo. Cuidado, conlleva dos significados básicos, ligados entre sí: el primero, con actitud de entrega hacia el otro; y el segundo, con el deseo afectivo de hacer algo por el otro. Desde épocas primitivas los cuidados, se han orientado a la atención de las necesidades de subsistencia: alimentación, vivienda, protección, recreación, confort, socializar, tener familia y distribuir el trabajo. En el saber cuidar tradicional predominaban las actividades preventivas, su valor era desde entonces considerado como don divino, era una necesidad de la que surgía una respuesta espontánea y voluntaria. Las normas se fueron desarrollando poco a poco en el seno de la familia. Cuidado también se refiere a asistir, que implica estar presente, comparecer, hacer compañía, auxiliar, socorrer, testimoniar, observar. Por ello cuidar incluye asistir, pero asistir no necesariamente incluye cuidar. Se puede estar presente, hacer compañía pero sin atender o esmerarse en lograr la recuperación de la salud o el bienestar del otro. La dedicación es fundamental para el cuidado. Cuando acaba la dedicación acaba el cuidado, El cuidado se desarrolla en un proceso de superar obstáculos y dificultades y la base es la dedicación, constituye el valor de la generosidad de quien lo aplica. El término de médico y medicar tiene su raíz en medicare y su significado original es atender. Los cuidados hoy incluyen a los procedimientos de: diagnóstico, tratamiento, rehabilitación o prevención y "el cuidado humanitario" buscando siempre hacer algo más.

b)- Los actores. Quienes interactúan en el cuidado, son: quien lo demanda y quien se dispone a ayudar. Integran una unidad al momento, intangible y virtual, que puede durar poco o largo tiempo. En este contexto, es apropiado llamar a esta relación social "Agente de Salud - Paciente o Persona" (AS-P). Es un concepto más profundo, aunque de otro contexto, es la Relación Médico Enfermera Paciente (RMEP). Descripciones de esta han dado lugar a magistrales obras de autores médicos, destacan españoles como Gregorio Marañon ${ }^{14}$ y Pedro Laín Entralgo, y mexicanos como Eduardo Césarman; ${ }^{15}$ Alberto Lipshitz; ${ }^{16}$ Ruy Pérez Tamayo. ${ }^{17}$ Hay otros muchos y buenos testimonios en la literatura, con elocuentes y extensos relatos o disertaciones. ${ }^{18}$ Bien sugieren que el tema es inefable. Pero el concepto ha evolucionado con la historia y no se detiene en su adaptación al progreso. Ignacio Chávez decía con toda razón "no hay mañana sin el ayer". Laín Entralgo advierte que la RMEP es una relación social creada entre dos personas, una diada. ${ }^{19}$ Las opiniones concuerdan en que esto funciona en la humanidad desde los tiempos más primitivos (ver tabla I). Surge espontáneamente y recibe diferentes denominaciones según el tiempo, la cultura, el estado del arte y los recursos en disponibilidad. 
Tabla I

\section{Síntesis evolutiva del conocimiento de cuidados}

\begin{tabular}{|c|c|c|c|c|}
\hline Etapas & $\begin{array}{c}\text { Primitiva de la } \\
\text { prehistoria y } \\
\text { barbarie }\end{array}$ & $\begin{array}{c}\text { Bíblica y religiones, } \\
\text { cristianismo }\end{array}$ & $\begin{array}{c}\text { Auge de las } \\
\text { ciencias y la razón }\end{array}$ & $\begin{array}{c}\text { Proyección } \\
\text { contemporáneo } \\
\text { El reto }\end{array}$ \\
\hline Desarrollo & $\begin{array}{c}\text { Primitivo, tradición y } \\
\text { magia }\end{array}$ & $\begin{array}{c}\text { Revelación divina } \\
\text { dogmas }\end{array}$ & $\begin{array}{c}\text { Las ciencias y el } \\
\text { desarrollo técnico. }\end{array}$ & $\begin{array}{c}\text { Innovación de la } \\
\text { ciencia y el } \\
\text { desarrollo social }\end{array}$ \\
\hline Conocimiento & $\begin{array}{c}\text { Intuitivo, atávico, } \\
\text { compasión humana. }\end{array}$ & $\begin{array}{c}\text { Religiones, } \\
\text { obediencia } \\
\text { irracional. }\end{array}$ & $\begin{array}{c}\text { Razonamiento } \\
\text { lógico, sobre los } \\
\text { hechos universales }\end{array}$ & $\begin{array}{c}\text { Dialéctica valórica y } \\
\text { búsqueda de } \\
\text { sentido. }\end{array}$ \\
\hline Estado del arte & $\begin{array}{c}\text { Natural, } \\
\text { primitivismo }\end{array}$ & $\begin{array}{c}\text { Espiritual, místico } \\
\text { el ascetismo }\end{array}$ & $\begin{array}{c}\text { Enfoque causal, la } \\
\text { enfermedad en el } \\
\text { individuo y la } \\
\text { población. }\end{array}$ & $\begin{array}{c}\text { La Evidencia, arte } \\
\text { interpretativo } \\
\text { Hermenéutica }\end{array}$ \\
\hline $\begin{array}{c}\text { Correlativo al } \\
\text { Agente - paciente }\end{array}$ & $\begin{array}{c}\text { Cualquier persona } \\
\text { hacia el que sufre }\end{array}$ & $\begin{array}{c}\text { Vocación personal y } \\
\text { espiritual, el buen } \\
\text { samaritano }\end{array}$ & $\begin{array}{c}\text { Profesionalismo, el } \\
\text { hospital, la Clínica } \\
\text { y Salud Pública. }\end{array}$ & $\begin{array}{c}\text { Atención a la Salud } \\
\text { Integral comunitaria }\end{array}$ \\
\hline Directriz de la \\
respuesta. & El Apoyo ante lo \\
sobrenatural & $\begin{array}{c}\text { Una confianza } \\
\text { frente a una } \\
\text { conciencia }\end{array}$ & $\begin{array}{c}\text { Atención personal } \\
\text { profesional y } \\
\text { especializado }\end{array}$ & $\begin{array}{c}\text { Equipo de Salud a } \\
\text { Comunidady } \\
\text { regulación sanitaria. }\end{array}$ \\
\hline
\end{tabular}

Dr. Rafael Chávez Domínguez 2010

Su naturaleza sigue una dinámica de cambio, ${ }^{20}$ como la vida misma. Lleva implícita una condición innata, la espiritual. ${ }^{21}$ Esta, le confiere todo y le da una identidad permanente. Esta relación es la esencia a destacar como "cuidado humanitario". Criterio alterno es tratar de apreciarlo bajo los preceptos de un nuevo conocimiento ${ }^{22} \mathrm{y}$ de la lógica, ${ }^{13}$ cabe considerarlos como elementos inherentes al cuidado. Le corresponde ser por una parte: el sujeto, ente, díada, RMEP o relación AS-P, una entidad invariable, pese a que cambien los personajes y su representación según el tiempo, espacio y circunstancias dadas; por otro lado, el objeto, el conjunto de cuidados, son la función de la relación AS-P, de naturaleza variable, destaca lo permanente, el cambio. Axiológicamente, se reconoce que ambos, sujeto y objeto interactúan, de modo que el objeto determina el valor real del sujeto. ${ }^{23}$ En otras palabras, el valor de la relación es por lo que resulta de ella. El cuidado humanitario, contiene ese valor universal, propio de la relación AS-.P, representa al conjunto del cual dependen muchas esperanzas. Los cuidados se han apreciado tradicionalmente como producto de la RMEP; pero están incluidos en la relación AS-P (Figura 1). Su interrelación consiste en que como sujeto, adquiere el valor de interactuar con el objeto, o sea de sus prácticas, los cuidados. Aquí, la unidad no cambia, sólo el cuidado y éste determina el valor humano que posee.

El carácter de los actores difiere al considerarlos separadamente, y también por eso reciben diversas denominaciones, que vale la pena recopilar. Por una parte: el cuidador, o el Agente de Salud, en contraste con el Agente causal (etiológico) de Enfermedad, es un término usado frecuentemente en el ámbito religioso, recuerda la gran preocupación, contribución y decidida participación permanente mantenida al respecto, por las organizaciones religiosas. ${ }^{24}$ Hay amplio testimonio de la vida religiosa en la salud en la historia de la humanidad. Pero antes, era ocupación de cualquier persona, un buen samaritano; un curandero o curador, brujo, chaman, un monje o el sacerdote. El concepto del Médico se formalizó en la Época de la Grecia Clásica, el desarrollo del conocimiento poco a poco lo vino consolidando como benemérita actividad profesional. También surge la participación de la Enfermería, de manera ejemplar y con formación profesional, cuyo propósito es el cuidado directo del paciente ${ }^{25,26}$ que ha identificado al cuidado como el objeto de estudio de la Disciplina. ${ }^{27}$ Más recientemente se han integrado técnicos y otras disciplinas afines que practican los cuidados y prolongan el papel del Agente de Salud, apoyándose en los conocimientos del arte y la ciencia médica. La contraparte de los actores: el paciente, que desde un principio ha sido el enfermo, por ser la persona portadora de una enfermedad (del Griego pathos: enfermedad). Sin 
embargo, hoy también incluye al no-enfermo, a cualquier persona o derechohabiente con riesgo de enfermar, es el cliente o el demandante de servicio y requiere ser mejor comprendido. El resultado es que la relación AS-P, siendo una imagen virtual e intangible, se hace presente en la mente de todos, gracias al sentido común que se intuye, la piedad, la misericordia, la clemencia, la compasión. También se advierte que el profesional de la salud que puede ser no-médico y el paciente ser no-paciente. Realmente, no significa que desaparezca la categoría en la relación AS-P, basta con aclarar y entender que se trata de una transición que se ilustra en la Tabla I. Al no comprenderse, ha creado confusión en el seno de la sociedad, aún entre profesionales. Ha surgido la idea de que la RMEP se ha acabado. Esto es un mal entendido que aquí se pretende aclarar. Lo que en efecto ocurre es que el enfermo pudiendo ser un no-enfermo, ante la presencia del médico, no siendo tan necesaria, crea un aparente absurdo que se disipa con la explicación plausible, ya que en presencia de un mal entendido así, lo demás es falso. La aclaración hay que difundirla y que se entienda que es por naturaleza cambiante. Lo perteneciente a la identidad es inmutable, y lo que evoluciona es el concepto de RMEP y AS-P. Ver Síntesis evolutiva de los cuidados.

\section{Relaciones del cuidado humanitario por la salud}

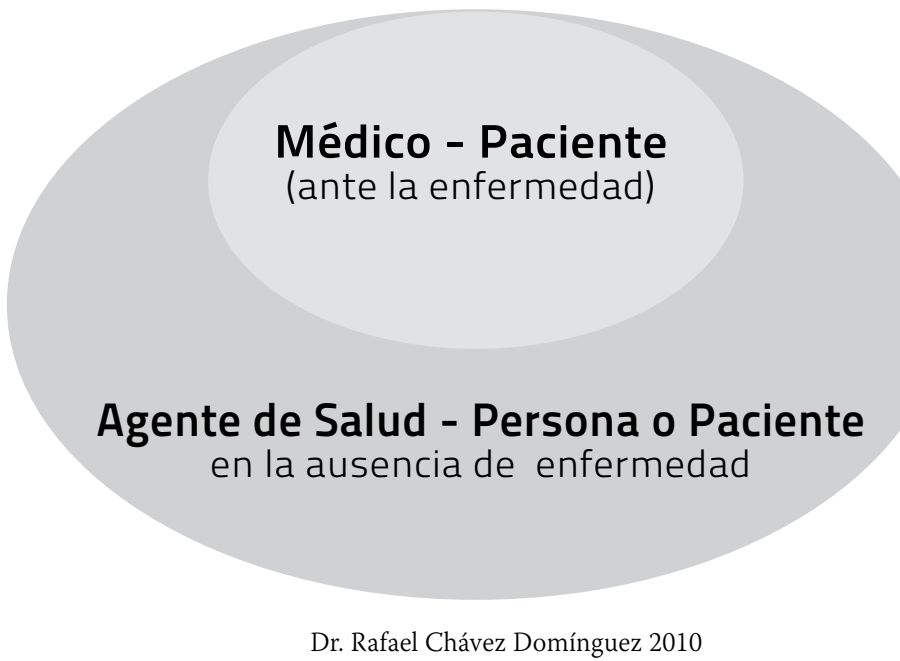

Figura 1

Tabla I.

Hay otras eventualidades que mencionar alrededor de la relación AS-P: la enfermedad. Su presencia es determinante, ésta genera la relación, tiene el carácter de necesaria y suficiente, pues en su ausencia o ante la duda, no hay paciente, ni enfermo, y tampoco exigencia de cuidados. La consideración de los Factores de Riesgo afecta a la relación AS-P, porque no son enfermedad, sino indicadores, con nexo de probabilidad causal y en muchos ámbitos sigue siendo motivo de confusión. El asunto trasciende, porque siendo no-suficiente, no genera la integración de relación AS-P y surgen las dificultades no esperadas, se pierde la autoridad necesaria. La difusión de los medios de comunicación han insistido en que es enfermedad, $y$ le hacen el centro de las actividades preventivas, consecuentemente, al no acatar consejos genera culpabilidad. En realidad, lo que el paciente o la población necesitan es comprensión y no distribución de culpa. Redunda en que la receptividad, por parte de una población confundida, no bien informada, incapaz de atender los mensajes, no genera el impacto deseado en la población, tras una aislada campaña publicitaria. Cualquier persona consciente, no puede darle crédito a la contradicción; y si el hecho se multiplica por los elementos de la comunidad, las acciones públicas o privadas fracasan. No siendo intención de las acciones preventivas, tiene que atender la confusión, sin que haya una fórmula práctica, universal, ni fácil de construir. Sin embargo, la acción conjunta, pensando y reflexionando, trae soluciones concretas, la visión integral 
favorece la creación apropiada del paradigma común.

c)- Los escenarios.

Las consideraciones ideales necesitan tomar en cuenta las realidades y sus tropiezos. Los cuidados se multiplican ante quienes los necesiten; pero también se suman quienes pudieran eventualmente necesitarlo. De todas maneras, propicia que cada día haya más pacientes, más procedimientos por realizar y aumenta la exigencia de recursos. Los sistemas administrativos se interponen pretendiendo ayudar en la distribución de los cuidados, o servicios; pero la rutina crea malos entendidos que llegan a desvirtuar su esencia. Estos medios altamente burocratizados debiendo ser el mejor aliado de la democracia, tienen preocupantes efectos adversos. La atención idónea de la salud es crear servicios ilimitados; pero los recursos para implementarla son finitos. De modo que, a las dificultades naturales para entregar los cuidados, hay que agregarles las inherentes a los intereses creados, incluyendo la misma regulación arbitraria de servicios que en el intento por lograr calidad y seguridad, choca con los rasgos indeseables y reclama mayor carga por otra atención permanentemente. ${ }^{28}$ La regulación de servicios, no siendo producto de las ciencias de la salud, sino de la administración e institucionalmente, del poder del Estado, encierra el peligro de ser impositiva con preocupantes consecuencias. La comprensión y el respeto a los fines del cuidado a la salud ayudan mucho, solo hay que tener cuidado de no confundir los medios de la burocracia, las metas de la organización ${ }^{29}$ y el fin de la misión por la Salud. El contrasentido afecta a la relación AS-P y pese a verla con optimismo para recuperar el sentido original, no resulta fácil. Es sorprendente ver la rapidez con que se polarizan los valores a lo económico y se contaminan de mezquindad y codicia. De no resolver este conflicto, surge el nuevo problema en los cuidados de la salud. La conciencia individual y la colectiva, no siempre se orientan a la visión humanitaria. Hasta llega a ser difícil hacer lo correcto. ${ }^{30}$ Destaca la importancia de recuperar el sentido positivo de las cosas. Pese a todo, deben prevalecer los fines, creados alrededor de los valores universales, fieles a la buena práctica, destinada por igual a todos, en todas las etapas de la vida e indistintamente en la salud y la enfermedad.

Al integrar los cuidados en la práctica se transforman gradualmente de Cuidado Personal a Trabajo de Equipo. En la línea del tiempo puede verse una transición (Tabla I) y ciertamente parece ser un camino natural. Lo que antes era labor personal, no deja de serlo, sólo se convierte en una acción organiza$\mathrm{da}$, justo para difundir mejor el saber y el hacer. El trabajo en equipo, mantiene su dimensión ontológica propia del ser, un proceso de relación reciproca entre confianza $y$ afectividad, de uno con otro ser humano "una confianza frente a una conciencia". Requiere de personas respetuosas consigo mismas y con la naturaleza, pues las acciones individuales no dejan de afectar al Ecosistema. Se exige respeto a la naturaleza, la Ecodulia, por ser el medio ecológico la fuente de la vida. La conducta en el grupo debe llegar a ser como la propia conducta individual. El trato digno con el paciente, es deseable que esté animado por los valores estéticos, realizado con arte, como se expresa en la música, la poesía, la pintura, la escultura, el sentido del humor, el baile, y otras formas, despierta emoción, respeto, lo justo del derecho y se alimentan el crecimiento espiritual. Ser profesional en el equipo de salud es, en primer lugar, ser persona, ${ }^{31}$ con calor humano. Quien brinda el cuidado con las manos y el corazón, lo transforma en algo valioso de afecto y cariño en beneficio del otro.

Ante las adversidades naturales cabe invocar resiliencia, la propiedad de un material para guardar memoria de la forma y recuperarla al cesar la fuerza deformante. Esta propiedad es aplicable a la relación AS-P, en defensa de sus intereses y en contra de los factores que la amenazan. Es prioritario mantener la identidad como propiedad privada. Las profesiones que tienen hoy la capacidad de comprender mejor esto, son la Enfermería y la Medicina por su vocación y preparación en este desempeño y por su investidura formal como Agente de Salud. El Ser: la relación AS-P. no puede cambiar. Lo que debe de cambiar es el modo de ser, o el modo de hacer 
las cosas: los cuidados.

La resiliencia ayuda a recuperar la forma, si se llegara a modificar. Es aplicable al Ser Agente de Salud. No puede cambiar su sentido positivo, la práctica del bien, no dañar a nadie, y defender la dignidad humana, la actitud apologética. El modo de ser y el modo de hacer en cambio, se refieren a hacer lo conducente, concientes de la diferencia que hay entre un "medio" para llevar a cabo el "fin" de esta noble misión.

El fin de los cuidados, es procurar salud y bienestar. Dos conceptos que van juntos y se complementan. Pero conviene hacer algunas aclaraciones que no se trata de fines últimos, sino de ideales cercanos. $\mathrm{Sa}$ $l u d$, persigue un ideal y señala con precisión la dirección y el sentido del proceder. Pero es un ideal y no lo real, que sólo se advierte al enfermar. Así, también cabe reflexionar que "una persona sana es un enfermo no diagnosticado". Significa que el sólo concepto de salud no basta, no puede motivar a quien presume de salud, ni a quien ha perdido todas sus facultades, un moribundo o paciente terminal. Igualmente, el cuidado de la salud nos preocupa hasta que ya hay daño y debiera preocupar desde antes, para tomar acciones preventivas, lo cual implica saber. Bienestar, en cambio, complementa el fin, tanto para un sano, como para un enfermo, o una persona en etapa terminal, en todos ellos se puede procurar bienestar, el cual es siempre bienvenido. Conviene precisar que no implica tener, poseer cosas y bienes sin importar lo que cuesten. El concepto incluye una legítima esperanza en el más allá de la muerte. Estos conceptos valen en todas las situaciones y son de incumbencia primaria al individuo, lo cual también es defendible como comunidad. El concepto se ha establecido desde los tiempos primitivos y de ninguna manera puede cambiar, ni ser objeto de transacciones comerciales, que ocurre bajo condiciones de conflictos de interés. Surge de la llana concepción en comunidad, y la sana relación AS-P lo representa. Las consideraciones económicas o financieras, son valores paralelos que tienen el propósito de garantizar la viabilidad, pero estos son un medio y no un fin.

\section{Las definiciones de Salud}

Los conceptos contenidos en las definiciones de salud sirven para acabar de comprender el fin, en la definición emitida por la oms cumple con sus fines operativos, pero no es una definición conceptual. Consiste en "un estado de completo bienestar físico, mental y social, y no solamente la ausencia de afecciones o enfermedades". Pero una definición conceptual es más amplia, no tiene límite. Se le puede describir como la suma de conceptos dispersos. Es lo que tiene sentido para una vivencia armoniosa, dichosa, responsable e interactiva con la herencia humana; incluye su realidad social, las condiciones de vida, la calidad de sus instituciones, la garantía del comportamiento personal en todas y cada una de las dimensiones de la persona, ya sea: física, emocional, intelectual, social, valoral y espiritual; ha de cubrir todas las etapas de la vida, relaciones personales y comunitarias, guardando equilibrio con la ecología, y ser el resultado de aceptar e integrar positivamente sus limitaciones, agravios y lo que llaman zonas oscuras de la existen- cia humana, es decir la enfermedad, la discapacidad, la agonía, la muerte y el duelo. Ha de posibilitar al hombre a alcanzar su autorrealización y plenitud humana.

\section{Concluyendo}

El cuidado humanitario es la meta de construir juntos un modelo unitario, algo por conocer con más intimidad a partir de la visión panorámica, algo nuevo o innovador que aprecia lo común de los cuidados particulares en la diversidad y está inmerso en el cuidado a la Salud. Es apoyo para no perder el sentido positivo en la conducta. Concilia la teoría y la práctica en bien de la prevención y la salud. Integrado en la relaciónAs-P es más universal y congruente con el proceso de cambio. El enfoque epistemológico puntualiza lo positivo y vigente acumulado por la historia. Lo pasado y útil no puede dejar de estar presente. No obstante lo complejo de las áreas y disciplinas que intervienen, su integración puede descubrir soluciones concretas y ser la llave maestra para compaginar las ciencias sociales, las ciencias humanas y las ciencias duras, con la participación de la tecnología, en apoyo a la preparación cultural, donde la educación es sólo parte y convoca a construir el paradigma común de las creencias del dominio popular. Es necesario invocarlo para superar las acciones ineficaces con desperdicio de recursos. La acciones preventivas en todos los niveles de atención ya sea en el hospital, la escuela, la comunidad el hogar, la cama de la unidad de cuidados intensivos y la Enfermería del futuro son el complemento necesario para cambiar el paradigma del cuidado reduccionista por el 
cuidado humanitario. Esto es lo que queda envuelto en la propuesta tomar en cuenta el amplio ámbito del Agente de Salud dedicado vocacional y profesionalmente al Cuidado Humanitario.

\section{REFERENCIAS BIBLIOGRÁFICAS.}

1 Organización Mundial de la Salud. Carta de Ottawa- OMS, 1986.

2 Colliere MF. Promover la Vida. Paris: Interediciones; 1982.

3 Rose G. Sick individuals and sick populations. International J. Epidemiol 1985; 14:32-38.

4 Zurita B, Lozano R, Ramírez T, Torres JL. Desigualdad en salud. Caleidoscopio de la Salud. 2003; 29-39. [Consultado 12 enero 2011] Disponible en: http://www. funsalud.org.mx/casesalud/caleidoscopio/01\%20Desigualdad.pdf

5 Borrel C, Palensia A, Pasarin I, Ortún $\mathrm{V}$. Widening social inequalities in mortality: the case of Barcelona, a southern European city. J Epidemiol Community Health 1997; 51(6):659-667.

6 Haaland Matlary J. Derechos humanos depredados. Hacia una dictadura del relativismo. Madrid: Ediciones Cristiandad; 2008. ISBN 9788470575358.

7 Popper K. La lógica de la Investigación Científica. Madrid: Tecnos; 1973.

8 Kuhn T. La estructura de las Revoluciones Científicas. México: Ed. Fondo de Cultura Económica 2007.

9 Martínez M. La Nueva Ciencia, Su desafío, Lógica y Método. México: Ed. Trillas; 1999.

10 Puskka P, Tuomilheto J, Nissinen A, Vartiainen E. The North Karelia Project. 20 Years Results and Experiences. Helsinki: National Public Health Institute KTL; 1995.
11 Benedicto XVI. Caritas in Veritate. Vaticano: Carta Encíclica; Junio 2009.

12 Chávez R, Ilarraza H. Metanoia y Bioética en la Prevencion Cardiovascular. En: Altamirano $\mathrm{M}$ y Cols. Editores. Dilemas Éticos en la Práctica Clínica. México: Corinter; 2009.

13 Gutierrez R. Introducción a la Lógica. México: Editorial Esfinge; 2008.

14 Jiménez Borreguero JF. Gregorio Marañón, el regreso del Humanismo. Fotoensayo biográfico. 2006, Editorial Egartorre. http:://gregoriomaranon.enarte.es//

15 Césarman E. Ser Médico. En: Siete Obras Escogidas. México: Ed. Porrúa; 1992.

16 Lifshitz A. La Relación Médico-Paciente en una Sociedad en Transformación. Acta Médica Grupo Angeles 2003. 1 (1): 59-66.

17 Pérez Tamayo R. Médicos y Medicina: El Caso Mexicano. Letras Libres. Febrero 2010

18 Hughes J. The Doctor-Patient Relationship: a review. [on line] Organization and Information at the Bed Side. Chapter One. Nov. 1994 [Consultado 5 marzo 2011] Disponible: http://www.changesurfer. com/Hlth/HuDiss.html

19 Laín Entralgo P. La relación médico-enfermo: Historia y teoría. Madrid, Alianza, 1983.

20 Barquín M. Historia de la Medicina. $8^{\text {a }}$. ed. México: Méndez Editores; 1998.

21 Lozano Barragán J. Mons. Teología y Medicina. México: Ed. Instituto Mexicano de Doctrina Social; 2000.

22 Hessen J. Teoría del Conocimiento. $13^{2}$. ed. Madrid: Espasa-Calpe; 1973.

23 Méndez JM. Razonamiento axiológico. En: José María Méndez. Cur- so Completo sobre Valores Humanos. Barcelona: Ed. PPU; 2007.

24 Alarcos FJ. Bioética y Pastoral de la Salud. Madrid: Ed. San Pablo 2002.

25 Watson J. Nursing: the Philosophy and Science of caring. Boston: Little Brown and Company, 1979.

26 Duran M. Dimensiones sociales, políticas y económicas del cuidado de enfermería. En: Grupo de cuidado. Dimensiones del cuidado. Colombia: Facultad de enfermería, Universidad Nacional de Colombia. 1998; pp 92-101.

27 Grupo de Cuidado. Avances conceptuales del Grupo de Cuidado. Colombia: Facultad de Enfermería. Universidad Nacional de Colombia: 1998; pp 8-16.

28 Donabedian A. La calidad de la atención médica. México: Ed. La Prensa Médica Mexicana 1984.

29 Chávez I. Profesión de Fe del Instituto de Cardiología de México. Libro Conmemorativo de 10 años de Fundación. México: Ed. INC. 1954. Citado también a los 20 años.

30 Chávez I. La moral médica frente a la medicina de nuestro tiempo. Humanismo Médico. Educación y Cultura. México: Ed. Colegio Nacional. 1978.

31 Watson J. Nursing: human science and human care, a theory of nursing. New York: National League for Nursing, 1988. 